Elsevier required licence: (C) <2017>. This manuscript version is made available under the CC-BY-NC-ND 4.0 license http://creativecommons.org/licenses/by-nc-nd/4.0/ 


\title{
Relating transition-state spectroscopy to standard chemical spectroscopic processes
}

\author{
Jeffrey R. Reimers ${ }^{\text {ab* }}$ and Noel S. Hush ${ }^{\text {cd* }}$ \\ a: Department of Physics and International Centre for Quantum and Molecular Structures, \\ Shanghai University, Shanghai 200444 China \\ b: School of Mathematical and Physical Sciences, University of Technology Sydney, NSW 2007 \\ Australia \\ c: School of Molecular Biosciences, The University of Sydney, NSW, 2006 Australia \\ d: School of Chemistry, The University of Sydney, Sydney, NSW 2006 Australia \\ * email: jeffrey.reimers@uts.edu.au,reimers@shu.edu.cn, noel.hush@sydney.edu.au
}

Keywords: adiabatic electron-transfer theory, quantum dynamics, Born-Oppenheimer breakdown, chemical entanglement, transition-state theory, non-adiabatic reactions, Born-Huang approximation

\begin{abstract}
Transition-state spectra are mapped out using generalized adiabatic electron-transfer theory. This simple model depicts diverse chemical properties, from aromaticity, through bound reactions such as isomerizations and atom-transfer processes with classic transition states, to processes often described as being "non-adiabatic", to those in the "inverted" region that become slower as they are made more exothermic. Predictably, the Born-Oppenheimer approximation is found inadequate for modelling transition-state spectra in the weak-coupling limit. In this limit, the adiabatic Born-Huang approximation is found to perform much better than non-adiabatic surfacehopping approaches. Transition-state spectroscopy is shown to involve significant quantum entanglement between electronic and nuclear motion.
\end{abstract}

\section{Highlights}

- transition-state spectra are calculated over a wide parameter region

- $\quad$ spectral and temporal responses may be simple or quite complex

- surface hopping methods fail to describe spectra usually classified as "non-adiabatic"

- transition-state spectroscopy embodies quantum entanglement 


\section{Introduction}

While transition states are involved in most chemical reactions, experimental methods for their direct observation remained elusive until the pioneering works of Zewail some 30 years ago [1-3]. A sense of the history of this search, the contributions of others, and the very wide ranging applicability of the results can be gauged from Polanyi and Zewail's 1995 review [4]. In modern times, this field is often called either "transition-state spectroscopy", as it involves vibrational and electronic spectroscopies performed at geometries near the transition state, or else "femtochemistry", owing to the small time period during which such geometries are sustained and the spectra of interest generated [5-13]. Starting with the initial spectral analysis of Volker, Metiu, Almeida, Marcus, and Zewail [14], the conceptual basis of femtochemistry has been seeded in the time-dependent understanding of spectroscopy pioneered by Heller [15-17]. Typically wavepackets corresponding to experimentally generated initial conditions [18] are followed and analysed, with, e.g., works by Tannor [19-25] and by Shapiro [26-32] showing how chemical control can be achieved, exploiting the non-ergodic nature of femtochemistry [14, 16, 33-35]. In general, the existence and possible role of quantum coherence in this non-ergodic motion remains a critical question [36-38]; quantum coherence requires much more than just ordered classical motion as the phase difference between quantum wavepackets propagating through equilibrium systems in thermal environments must also be maintained, generating quantum entanglement [39]. Not just transition states are now studied by also photochemical species existing down to ultrashort timescales with wide ranging applications in energy harvesting and catalysis, as revealed, e.g., by the works of Kobayashi [40-50].

Not all chemical phenomena involve transition states, however. Non-radiative transitions and avoided crossings also give rise to ultrafast processes and can equally be studied using transition-state spectroscopic techniques [51-56]. Such transitions can arise either because the coupling between diabatic states goes to zero at a conical intersection, or else because the transitionstate becomes converted into a point of inflection on the potential-energy surface of a highly exothermic (or endothermic) reaction. The later scenario is called the "inverted" region of chemical kinetics [57]. However, transition states can also disappear when tautomers are replaced by “delocalized” resonance hybrids [58, 59], providing another asymptotic limit of transition-state spectroscopy.

The transition-state spectroscopy of non-dissociative reactions, as well as the spectroscopy of non-adiabatic, inverted, and delocalized processes, are all describable using the adiabatic electron transfer developed by Hush initially to describe electron-transfer and atom-transfer reactions [5866]. This approach has recently been generalized to include all types of isomerization processes and reactions between bound species, including treatment of aromatic chemistry [67]. Achievements include the first explanation as to why $\mathrm{NH}_{3}$ is tetrahedral whilst $\mathrm{PH}_{3}-\mathrm{BiH}_{3}$ are octahedral [68], understanding properties of Born-Oppenheimer breakdown as well as the calculation methods used to examine it [69], and modelling the entanglement developed during chemical reactions [70, 71]. Here, we consider key results predicted for transition-state spectroscopy and related non-adiabatic processes, focusing on how it relates to other chemical spectroscopies. 


\section{The basic model used in adiabatic electron-transfer theory.}

In its simplest analytical form as applied here, adiabatic electron-transfer theory assumes parabolic surfaces and includes only one type of nuclear motion, motion along the reaction coordinate. These assumptions lead to three limitations in its applicability to transition-state spectroscopy: i) often motions other than the reaction coordinate are critical to observed phenomena [4, 72-74], including continuous motions like solvent friction [75]; ii) conical intersections intrinsically involve at least two nuclear coordinates; and iii) many reactions of interest involve bimolecular processes that cannot easily be discussed using harmonic potentials. However, the basic model does provide an underlying basis for critical aspects of these more complicated phenomena. In particular, the connection between chemical bonding and transition-state spectroscopy [76] can be discussed, and its inherent quantum density-matrix based description links smoothly to modern more thorough approaches [77].

\section{2a. Diabatic descriptions of chemical and spectroscopic processes.}

In its original formulation, adiabatic electron-transfer theory expresses chemical structure, thermodynamics, kinetics, and spectroscopy in terms of two coupled harmonic diabatic potentialenergy surfaces. Diabatic surfaces were first used to discuss transition states, avoided crossings, and non-adiabatic reactions by London [78, 79] and quickly applied by Eyring and Polanyi to produce the analytical LEP potential-energy surface [80] for general triatomic molecules [81], as well as by Horiuti and Polanyi [82] for proton transfer reactions and Wall and Glockler [83] for isomerization reactions. These early approaches were often based on Morse diabatic functions that facilitated bond dissociation, with Hush [84] later introducing the simpler harmonic surfaces for bound reactions. The basic equations can be written in three formally equivalent forms, two of which are that are diabatic with the other being adiabatic (see later). Each representation forms simple descriptions of some observed phenomena but complex descriptions of other phenomena, and while accurate numerical solutions yield the same answers, the degree of difficulty in obtaining accurate solutions varies accordingly [67, 69, 85, 86]. For studying chemical kinetics and transition states, the most common diabatic representation used was introduced by Hush [60-62, 84] and is known as the localized diabatic description, formally represented by the Hamiltonian

$$
\mathbf{H}^{\mathbf{L}}=\left[\begin{array}{cc}
T+\frac{\hbar \omega}{2}\left(Q+Q_{\mathrm{m}}\right)^{2}+J-\frac{\lambda}{4} & J \\
J & T+\frac{\hbar \omega}{2}\left(Q-Q_{\mathrm{m}}\right)^{2}+J-\frac{\lambda}{4}+E_{0}
\end{array}\right]
$$

where $\omega$ is the harmonic vibration frequency, $Q$ a dimensionless normal coordinate expressed in terms of the zero-point length $\sqrt{\hbar / \omega}$ [87] that describe the reaction coordinate, the equilibrium geometries of the reactants and products diabatic surfaces are located at $Q=-Q_{\mathrm{m}}$ and $Q=Q_{\mathrm{m}}$, respectively, $T=-\frac{\hbar \omega}{2} \frac{\partial^{2}}{\partial Q^{2}}$ is the nuclear kinetic energy operator, $\lambda=2 \hbar \omega Q_{\mathrm{m}}^{2}$ is the reorganization energy, $E_{0}$ is the energy difference between products and reactants, and $J$ is the electronic coupling (resonance energy) linking the reactant to the product. The basis set for this Hamiltonian are two localized diabatic electronic functions $\left\{\psi_{\mathrm{R}}, \psi_{\mathrm{P}}\right\}$. Diabatic representations are not unique, however, and a second commonly used representation is obtained by a $45^{\circ}$ rotation [86] of these 
functions to the delocalized diabatic basis $\left\{\psi_{\mathrm{G}}, \psi_{\mathrm{T}}\right\}$ with $\psi_{\mathrm{G}}=\left[\psi_{\mathrm{R}}-\psi_{\mathrm{P}}\right] / 2^{1 / 2}$ and $\psi_{\mathrm{T}}=\left(\psi_{\mathrm{R}}+\psi_{\mathrm{P}}\right) / 2^{1 / 2}$, producing

$$
\mathbf{H}^{\mathbf{D}}=\left[\begin{array}{cc}
T+\frac{\hbar \omega}{2} Q^{2}+\frac{E_{0}}{2} & \alpha Q-\frac{E_{0}}{2} \\
\alpha Q-\frac{E_{0}}{2} & T+\frac{\hbar \omega}{2} Q^{2}+2 J+\frac{E_{0}}{2}
\end{array}\right]
$$

where $\alpha=\hbar \omega Q_{\mathrm{m}}$ is a linear vibronic-coupling constant. Expressed in this form, adiabatic electrontransfer theory is often called the pseudo Jahn-Teller effect [88], providing a traditional way for considering molecular spectroscopy. The labels $\mathrm{G}$ and $\mathrm{T}$ stand for the ground state and its "twin" state and describe diabatic spectroscopic states rather than reactants or products, the type of labels usually applied in considering aromatic and other spectroscopies. The twin state of the ground state is an excited state that has properties directly related to those of the ground state- knowing one state is tantamount to knowing the other. This concept was first introduced by Shaik [89-93] but he thought the twin state to always by a state formed by single excitation from the ground state. Indeed, such singly excited states are the twin states for electron transfer problems, forming the basis of intervalence charge-transfer (IVCT) spectroscopy [64], but in general the twin state is actually the state of highest-possible excitation amongst the frontier orbitals [67]. Recognizing the actual nature of the twin state, and how other states like single-excitation states relate to it and to the ground state, allows adiabatic electron-transfer theory to be immediately generalized to isomerization reactions and aromatic process modelling, completing the vision of quantum chemistry pioneers like London, Eyring, and Polanyi.

\section{2b. Adiabatic representation.}

Both diabatic descriptions lead without approximation to the same adiabatic description of the chemical and spectroscopic properties of the system. This is obtained by applying the BornOppenheimer transformation to either $\mathbf{H}^{\mathbf{L}}$ or $\mathbf{H}^{\mathbf{D}}$, diagonalizing these at each individual nuclear coordinate $Q$ to create $[69,94,95]$

$$
\mathbf{H}^{\mathbf{B O}}=\left[\begin{array}{cc}
T+\varepsilon_{-}(Q)+\Delta H^{D C}(Q) & \Delta P^{F D}(Q) \frac{\partial}{\partial Q}+\Delta H^{S D}(Q) \\
\Delta P^{F D}(Q) \frac{\partial}{\partial Q}+\Delta H^{S D}(Q) & T+\varepsilon_{+}(Q)+\Delta H^{D C}(Q)
\end{array}\right],
$$

where $\varepsilon_{-}(Q)$ and $\varepsilon_{+}(Q)$ are the eigenvalues [59] of $\mathbf{H}^{\mathbf{L}}$ and $\mathbf{H}^{\mathbf{D}}$

$$
\varepsilon_{ \pm}(Q)=\frac{E_{0}}{2}+\frac{\hbar \omega}{2} Q^{2} \pm\left[\left(\frac{E_{0}}{2}-\hbar \omega Q_{\mathrm{m}} Q\right)^{2}+J^{2}\right]^{1 / 2}
$$

and the three correction terms to the Born-Oppenheimer approximation to Eqn. (3) [79, 94, 95 , 96, 97]

$$
\mathbf{H} \approx\left[\begin{array}{cc}
T+\varepsilon_{-}(Q) & 0 \\
0 & T+\varepsilon_{+}(Q)
\end{array}\right]
$$

are [69] the momentum (or first derivative) correction 


$$
\Delta P^{F D}(Q)=\frac{-\hbar^{2} \omega^{2} Q_{\mathrm{m}} J}{2 J^{2}+\hbar \omega \lambda\left(Q-Q_{\mathrm{x}}\right)^{2}}=\frac{\hbar \omega}{2} \frac{Q_{\mathrm{C}}}{Q_{\mathrm{C}}^{2}+\left(Q-Q_{\mathrm{x}}^{2}\right)},
$$

the diagonal correction

$$
\Delta H^{D C}(Q)=\frac{1}{2 \hbar \omega}\left(\Delta P^{F D}(Q)\right)^{2}
$$

and the kinetic energy (or second derivative) correction

$$
\Delta H^{S D}(Q)=\frac{2}{\hbar \omega} \frac{Q-Q_{\mathrm{x}}}{Q_{\mathrm{C}}}\left(\Delta P^{F D}(Q)\right)^{2}
$$

where $Q_{\mathrm{x}}$ is the coordinate of intersection of the localized diabatic surfaces $[61,62]$

$$
Q_{\mathrm{x}}=\frac{E_{0}}{\lambda} Q_{\mathrm{m}}
$$

and

$$
Q_{\mathrm{C}}=\frac{2 J}{\lambda} Q_{\mathrm{m}},
$$

gives the Born-Oppenheimer cusp diameter $\left|Q_{c}\right|$ [69]. The electronic basis set $\left\{\left|\psi_{-}\right\rangle,\left|\psi_{+}\right\rangle\right\}$used to express $\mathbf{H}^{\mathbf{B O}}$ is obtained from the eigenvectors of $\mathbf{H}^{\mathbf{L}}$ (or else an equivalent expression obtained by diagonalizing $\mathbf{H}^{\mathbf{D}}$ ) as

$$
\begin{aligned}
& \left|\psi_{-}\right\rangle=a(Q)\left|\psi_{\mathrm{R}}\right\rangle+b(Q)\left|\psi_{\mathrm{P}}\right\rangle \\
& \left|\psi_{+}\right\rangle=-b(Q)\left|\psi_{\mathrm{R}}\right\rangle+a(Q)\left|\psi_{\mathrm{P}}\right\rangle
\end{aligned},
$$

where [59]

$$
\begin{gathered}
a^{2}(Q)=\frac{1}{2}+\frac{\frac{E_{0}}{\lambda}-\frac{Q}{Q_{m}}}{2\left[\left(\frac{E_{0}}{\lambda}-\frac{Q}{Q_{m}}\right)^{2}+\left(\frac{2 J}{\lambda}\right)^{2}\right]^{1 / 2}}, \text { and } \\
b^{2}(Q)=\frac{1}{2}-\frac{\frac{E_{0}}{\lambda}-\frac{Q}{Q_{m}}}{2\left[\left(\frac{E_{0}}{\lambda}-\frac{Q}{Q_{m}}\right)^{2}+\left(\frac{2 J}{\lambda}\right)^{2}\right]^{1 / 2}}=1-a^{2}(Q) .
\end{gathered}
$$

The coefficients $a$ and $b$ are used to specify the density matrix of the system, $b^{2}(Q)$ specifies the product component of the adiabatic state at each nuclear coordinate, with $b^{2}\left(Q_{\mathrm{X}}\right)$ approximating 
the product character of the transition state [61, 62, 79, 97]. The cusp diameter is so named as transformation of the diabatic Hamiltonians into the adiabatic basis introduces a pitchforkbifurcation cusp catastrophe [98-101] that manifests derivative discontinuities in $\varepsilon_{ \pm}(Q)$ whenever $J=0$. For finite $J$, the nature of the lower surface changes from largely reactant-like to largely product-like when the coordinates change by $Q_{\mathrm{C}}$ near the crossover point $Q_{\mathrm{X}}[61,62,79,97]$. During this changeover, the transition-state region is crossed in a process well recognized in the Reaction Force model of chemical reactivity [102-105]. The emphasis on the crossover region as the critical element of general electron-transfer processes sets adiabatic electron-transfer theory apart from its alternative instantaneous-transition alternatives [106], focusing on the properties of the transition region and the charged transferred at all points along the reaction coordinate, allowing for Zewail's observation of transition-state spectroscopy.

2c. Occurrence of a transition state on the lower Born-Oppenheimer surface.

The lower Born-Oppenheimer adiabatic surface $\varepsilon_{-}(Q)$ shows a transition state whenever [69]

$$
\left(\frac{E_{0}}{\Delta E}\right)^{2}<\left[1-\left(\frac{2|J|}{\lambda}\right)^{2 / 3}\right]^{3}\left[1+\left(\frac{2 J}{\lambda}\right)^{2}\right]
$$

where $\Delta E=\left(4 J^{2}+\lambda^{2}\right)^{1 / 2}$ is the electronic energy gap. In the weak-coupling limit of $2|J| / \lambda \ll 1$, this reduces to

$$
\left|E_{0}\right|<\lambda
$$

and is referred to the normal region of chemical kinetics; when no transition state exists it is referred to as being the inverted region, an important scenario championed by Marcus [57, 106]. When there exists a transition state, the barrier height in the weak-coupling limit be approximated by the energy of the localized diabatic states at their crossing point $Q=Q_{\mathrm{x}}$ as $[61,107]$

$$
\Delta E^{\ddagger}=\frac{\left(\lambda+\Delta E_{0}\right)^{2}}{4 \lambda},
$$

a quantity also taken to be indicative of system properties even in the inverted region and treated if indeed a transition state did exist. For general symmetric reactions with $\Delta E=0$, transition states exist whenever [58, 59, 108]

$$
\frac{2|J|}{\lambda}<1
$$

This is known as the localized regime, with the alternative being the delocalized regime. Resonance stabilized aromatic molecules like benzene are examples of systems in the delocalized regime, with its hypothetical Kekulé “reactant” and "product” structures not appearing as local minima on $\varepsilon_{-}(Q)$ [67]. In the localized regime the barrier height is given by [69] 


$$
\Delta E^{\ddagger}=\frac{(\lambda-2|J|)^{2}}{4 \lambda} .
$$

The parameter space over the whole model in which $\varepsilon_{-}(Q)$ shows a transition state (Eqn. (14)) is shown in Fig. 1. The barrier height is in general close to [69]

$$
\Delta E^{\ddagger} \approx \frac{\left(\lambda+E_{0}-2|J|\right)^{2}}{4 \lambda} .
$$

\section{2d. The Born-Huang adiabatic approximation.}

The Born-Oppenheimer approximation Eqn. (5) is not the only way to simplify Eqn. (3) into an adiabatic form, an alternative being the Born-Huang approximation [79, 94, 95 , 96, 97]

$$
\mathbf{H} \approx\left[\begin{array}{cc}
T+\varepsilon_{-}(Q)+\Delta \mathrm{H}^{D C}(Q) & 0 \\
0 & T+\varepsilon_{+}(Q)+\Delta \mathrm{H}^{D C}(Q)
\end{array}\right]
$$

Here, the diagonal correction $\Delta H^{D C}(Q)$ to the Born-Oppenheimer approximation from Eqn. (7) is retained whilst the non-adiabatic coupling terms $\Delta P^{F D}(Q)$ and $\Delta H^{S D}(Q)$ that couple the ground and excited adiabatic states remain neglected. From Eqns. (6)-(7), the effect of the diagonal correction is to add within the intersection region at $Q=Q_{\mathrm{x}}$ a spike of width $Q_{\mathrm{C}}$ and height

$$
\Delta \Delta E^{\ddagger}=\left(\Delta H^{D C}(Q)\right)_{\max }=\frac{\hbar \omega}{8 Q_{\mathrm{C}}^{2}}
$$

in parallel to both Born-Oppenheimer surfaces. In the delocalized region its contribution is negligible as $Q_{\mathrm{C}}$ becomes wider than the length scale of chemical interest and $\Delta \Delta E^{\dagger}$ becomes much less than both the electronic and vibrational energy scales of the system. However, in the weak-coupling limit the DC spike becomes much higher than the energy $\Delta E^{\ddagger}$ at the crossover point. As a result, the lower Born-Huang adiabatic surface almost always manifests a transition state, making Zewail's concept of transition-state spectroscopy directly relevant to most electron-transfer reactions performed in the inverted region.

\section{2e. Failure of the Born-Oppenheimer approximation.}

Using the Born-Oppenheimer description, processes in the inverted region are usually described as being non-adiabatic as they involve radiationless transitions between the two different potential-energy surfaces. The reactant and product surfaces do not intersect and so there is no transition state available to facilitate the reaction. Typically, however, use of the alternate BornHuang adiabatic description typically re-creates a transition state, with the reaction then describable as occurring via nuclear tunneling through the high but narrow DC spike [69], facilitating electron transfer. In the weak-coupling limit, failure of transition-state theory based on the BornOppenheimer approximation to describe electron-transfer reactions is usually expressed in terms of the Levich-Dogonadze rate equation [109-112] 


$$
k=\frac{2 \pi J^{2}}{\hbar \sqrt{4 \pi \lambda k_{\beta} T}} \exp \frac{-\Delta E^{\ddagger}}{k_{\beta} T}=\kappa \frac{\omega}{2 \pi} \exp \frac{-\Delta E^{\ddagger}}{k_{\beta} T},
$$

where $\kappa$ is called the transmission coefficient,

$$
\kappa=\pi^{3 / 2}\left(\frac{\lambda}{k_{\beta} T}\right)^{1 / 2} Q_{c}^{2}
$$

The maximum physically meaningful value for $\kappa$ is unity, with perceived values greater than this indicating that the weak-coupling approximation used by Levich and Dogonzdze no longer holds. When $\kappa=1$, Born-Oppenheimer-based transition state theory is adequate to describe the reaction. However, instead of viewing $\kappa$ as specifying a radiationless transition rate between the two localized diabatic surfaces or else $1-\kappa$ as specifying a radiationless transition rate between the Born-Oppenheimer adiabatic surfaces, similar results can be obtained using the Born-Huang approximation [69] for the ground state without the need to include the second surface.

A common method used for understanding radiationless transitions in general chemical reactions involves simulating surface hopping between Born-Oppenheimer surfaces [113, 114], driven typically by only the non-adiabatic coupling term $\Delta P^{F D}(Q)$. In this approach, the diagonal correction is usually ignored as transition rates depend on the relative slopes of the two surfaces, a quantity which $\Delta H^{D C}(Q)$ does not modify. However, application of only the Born-Huang approximation usually works much better than this approach [69]. Surface hopping based on $\Delta P^{F D}(Q)$ is naively appealing as this correction appears at lower order in $1 / Q_{\mathrm{C}}$ than does $\Delta H^{D C}(Q)$ or $\Delta H^{S D}(Q) . \Delta H^{D C}(Q)$ does not change relative slopes but it does control what nuclear configurations can be accessed near conical intersections and so actually does have a profound effect. Further, the chaotic behavior of dynamics near a cusp means that perturbation treatments are inappropriate [61, 62, 79, 97] and hence all three Born-Oppenheimer correction terms must be applied in accurate calculations [69].

\section{Applications based on accurate numerical solutions of spectroscopic properties}

We investigate the spectra expected for electron-transfer processes happening near the transition state. A total of 16 examples from the model parameter space are considered, marked (a)(p) on Fig. 1. These are ran at values of $2 J / \lambda=0.03$ (weak coupling typical of electron transfer), 0.3 (intermediate coupling with orthodox transition states), 0.8 (borderline delocalized akin to ammonia inversion and the Creutz-Taube ion [67]), and 1.5 (delocalized). Also used are $E_{0} / \Delta E=$ 0.25 (endothermic reaction), 0 (symmetric reaction), -0.25 (exothermic reaction) and -1.1 (invertedregime highly exothermic reaction). The model contains 4 parameters $J, \lambda, E_{0}$, and $\omega$ but one of these can always be thought of as simply an energy scaling. We use $2 J / \lambda$ and $E_{0} / \Delta E$ as characteristic parameters, and a third can be expressed as $\hbar \omega / \Delta E$ which, in these trajectories, is constrained to be 0.1 throughout. As $\hbar \omega / \Delta E$ approaches 1 it is likely that Jahn-Teller effects associated with conical intersections will contribute significantly to observed processes, making any one-dimensional model inappropriate. Smaller values than 0.1 represent systems with deep wells supporting many bound vibrations, but no qualitative changes in behaviour are expected and so this 
dimension is not explored herein. Basic properties of the 16 systems considered are summarized in Table 1, including the activation energy (using the crossover energy approximation Eqn. (16) in the inverted regime), the Born-Huang spike height (Eqn. (21)) and the cusp diameter (Eqn. (10)).

3a. Representation of the dipole operator specifying electronic and vibrational transitions.

To determine spectra, the electric dipole operator of the system needs to be specified for each electronic basis set. In the localized diabatic basis, the dipole operator is assumed to be

$$
\mathbf{M}^{\mathbf{L}}=\frac{R}{2}\left[\begin{array}{cc}
-1 & 0 \\
0 & 1
\end{array}\right]
$$

where $R$ is the distance through which the active electrons shift during the reaction, a form that is highly appropriate to electron-transfer problems as this is then just the distance through which the electron is transferred [86, 115-117]. In the delocalized diabatic basis, this operator becomes

$$
\mathbf{M}^{\mathbf{D}}=R\left[\begin{array}{ll}
0 & 1 \\
1 & 0
\end{array}\right]
$$

a form that is highly appropriate to understanding the intense electronic transitions observed in aromatic molecular spectroscopy. In the Born-Oppenheimer basis, the dipole operator becomes coordinate dependent:

$$
\mathbf{M}^{\mathbf{B O}}(Q)=\frac{R}{2}\left[\begin{array}{cc}
-\left(a^{2}(Q)-b^{2}(Q)\right) & 2 a(Q) b(Q) \\
2 a(Q) b(Q) & a^{2}(Q)-b^{2}(Q)
\end{array}\right]
$$

When applied using the Born-Oppenheimer or Born-Huang approximations, the diagonal elements of this matrix generate traditional infrared vibrational spectra and associated (perhaps microwave) vibrational tunneling transitions, whilst the off-diagonal terms facilitate Franck-Condon and Herzberg-Teller (etc.) electronic transitions.

All results shown in Fig. 2 and later are fully converged numerically and hence are independent of the actual Hamiltonian and corresponding dipole-moment operator used. However, in addition we also show spectra obtained using the approximation to Eqn. (26):

$$
\frac{R}{2}\left[\begin{array}{cc}
0 & 2 a(Q) b(Q) \\
2 a(Q) b(Q) & 0
\end{array}\right] .
$$

The full Hamiltonians all embody mixing between vibrational and electronic motions and so result in spectra that can differ somewhat from basic expectations. Transition-state spectroscopy can also investigate low-energy electronic processes of the same order as vibrational energy spacings, further confusing the distinction between processes considered to be either primarily electronic or primarily nuclear in origin. As a result, spectral line intensities can simultaneously gain strength from both the diagonal elements and the off-diagonal elements of the dipole matrix, with the two effects interfering with each other as they sum to give line transition moments. The approximation in Eqn. (26) includes only the electronic transition component to the total intensity, facilitating the identification of electronic spectra, vibrational spectra, and any interferences between them. In Fig. 2 , the total cross-section is shown in blue whilst its electronic component is shown in red, giving the 
allusion of magenta colouring whenever the electronic and vibrational contributions do not interfere and the total spectrum is dominated by just its electronic component.

\section{3b. Time-dependent representation of molecular spectra and properties.}

As the time spent in the vicinity of a transition state is usually limited, time-dependent methods provide the natural way for considering transition-state spectroscopy. In these, initial wavepackets $|\psi(0)\rangle$ are constructed and their time dependence followed, with the spectra calculated from this. In this way, the spectra depend explicitly on the initial wavepacket chosen. In standard equilibrium spectroscopy, the initial wavepacket is often just the stationery state of the system identified as the ground vibrational level of the ground electronic state, though this may be thermally weighted if necessary [17]. Here we consider simple wavepackets that would describe the ground-state of the diabatic surfaces should they be centred on the diabatic potential minima, but their initial positions are chosen depicting wavepacket energies in the vicinity of the transition state. It is possible that in experiments such initial wavepackets could be created from either some external spectroscopic process or else following reaction from some initial chemical species. Under these initial conditions they are not eigenstate of any potential and hence evolve in time according to the time-dependent Schrödinger equation specified by Hamiltonian $\mathbf{H}$ expressed in the same basis (diabatic or adiabatic) as is the wavepacket itself, making $|\psi(t)\rangle=e^{-i \mathbf{H} t / \hbar}|\psi(0)\rangle$. In general [15-17], the absorption and emission of light associated with the wavepacket's motion is given as the Fourier transform

$$
\sigma(\omega)=\int_{-\infty}^{\infty} c(t) e^{i \omega t} \mathrm{~d} t
$$

of the autocorrelation function

$$
c(t)=\left\langle\psi(0)\left|e^{i \mathbf{H} t / \hbar} \mathbf{M} e^{-i \mathbf{H} t / \hbar} \mathbf{M}\right| \psi(0)\right\rangle .
$$

Calculated spectral cross-sections $\sigma(\omega)$ are shown for example in Fig. 2, with associated absorption spectra proportional to $\omega \sigma(\omega)$ whilst emission spectra are proportional to $\omega^{3} \sigma(-\omega)$.

The origins of transition-state spectra like that show in Fig. 2 can be described in terms of the properties of the wavepacket dynamics $|\psi(t)\rangle$ itself, with that corresponding to Fig. 2 shown in Figs. 3 and 4 for model scenarios (a)-(h) and (i)-(p), respectively. Whilst the use of different electronic basis states, either diabatic or adiabatic, leads for converged calculations to the same wavepacket dynamics and spectra, the different basis sets represent this information in different ways and facilitate use of different approximations describing the spectra. So while Fig. 2 shows only spectra calculated using $\mathbf{H}=\mathbf{H}^{\mathbf{B O}}$ (Eqn. (3)), labelled in Figs. 3 and 4 as the "full" calculation (FC), the later figures also shows formally equivalent results labelled (L) obtained using the localized diabatic Hamiltonian $\mathbf{H}=\mathbf{H}^{\mathbf{L}}$ (Eqn. (1)). The dynamics expressed in terms of the localized diabatic reactant and product surfaces is shown using red and blue colouring to indicate how much of the wavepacket is on each diabatic surface at any point in time. In parallel, the composition of the wavepacket projected onto the ground-state and excited-state BornOppenheimer surfaces is shown in green and magenta, respectively. Here, the initial wavepackets 
are located purely on the Born-Oppenheimer ground-state surface and are mixtures of the reactant and product surfaces, but these natures evolve during the trajectories.

\section{3c. Numerical solution method.}

A wide variety of methods are available for evaluating Eqn. (29). In principle, the dynamics can be solved using explicit time-dependent methods that integrate the differential equation specified by the time-dependent Schrödinger equation, something that can often be approximated accurately by classical or semiclassical means. In this way solutions can be obtained for large systems using full Hamiltonians rather than simple model ones like our Hamiltonians. However, dynamics around conical intersections is intrinsically chaotic and hence converging such approaches to the desired accuracy is always difficult, especially in the weak-coupling limit in which $\kappa$ gets small. We use a method that maximizes convergence but is of high cost, as can be done typically only for simple model Hamiltonians. The full details are described in detail elsewhere [69]. Basically, a harmonic-oscillator vibrational basis is used for each electronic basis state centred at $Q=0$. This is truncated at 256 levels per state, sufficient for all examples considered herein to achieve full convergence of even the poorly convergent calculations (required computer time proportional to $Q_{c}^{-6}[69]$ ) performed using the Born-Oppenheimer electronic basis. All eigenstates of the appropriate Hamiltonian are then obtained by full matrix diagonalization, allowing an analytical representation of the time dependence of the initial wavepacket that gives stable solutions throughout chaotic domains. A mixture of analytically determined and numerically evaluated matrix elements is used to construct the Hamiltonians, with no convergence issues arising.

\section{3d. Results for trajectories starting in the reactant domain.}

The example trajectories and spectra previously mentioned shown in Figs. 2-4 were obtained from trajectories starting far on the reactant side of the transition state. Also, the initial wavepackets $|\psi(0)\rangle$ are constructed on the lower Born-Oppenheimer surface and have the shape of the ground-state vibrational wavefunction of each isolated diabatic potential. In Figs. 3 and 4, the wavefunction is sketched on top of the appropriate potential-energy surface(s) at time $t=0$, $t=\pi / 3 \omega, \quad t=\pi / \omega, t=4 \pi / 3 \omega, t=5 \pi / 3 \omega$, and $t=2 \pi / \omega$. The transition-state region is accessed at a time near $\pi / \omega$, whilst one recurrence of the wavepacket occurs at around time $2 \pi / \omega$.

Collected in Table 1 are a variety of properties summarizing key features of the calculated dynamics. The transmission coefficient $\kappa_{2 \pi}$ (Eqn. (22)) indicates the fraction of the wavepacket transferred from reactants to products after 1 vibrational period. When large, this indicates that classical transition-state theory is adequate in describing reaction kinetics. Note, however, that it is always short of unity as the quantum wavepackets embodies classical states both slightly above and below any classical transition-state barrier, so some degree of non-reaction is always expected. Also, $f_{\pi}^{+}$gives the fraction of the wavepacket transferred to the excited adiabatic state after half of a period, crudely indicating the significance of non-adiabatic dynamical processes. Finally, Table 1 also includes the quantum entanglements developed between the electronic and nuclear motions after one period of vibration, projected onto the localized diabatic states, $S_{2 \pi}^{L}$, as well as onto the Born-Oppenheimer electronic states, $S_{2 \pi}^{B O}$. The projection onto the localized diabatic states is in- 
principle experimentally measurable using spatially resolved detectors and could be utilized in some quantum information-processing device [71, 118-120], whilst that revealed in the BornOppenheimer basis provides a unique and sensitive way of understanding the failure of the BornOppenheimer approximation [70].

The calculated spectral densities (a)-(d) in the weak-coupling limit with $2 \mathrm{~J} / \lambda=0.03$ depict vibrational tunnelling transitions from the reactant well to the product well. While these transitions are nominally considered to be vibrational, because of the electron-vibration coupling, these pull the electron with them to facilitate electron transfer if unconsidered factors induce decoherence [121]. All states contained in the wavepacket contribute to this tunnelling. The transmission factors are all very low for (a)-(c) $(0.03-0.04)$ but the initial wavepacket for (d) already has a significant overlap with the product state and so the tabulated measure $\kappa_{2 \pi}$ is poorly indicative of reactivity. Similarly, the other tabulated properties become difficult to interpret and henceforth we discuss only (a)-(c). The quality of the Born-Oppenheimer adiabatic description of the reaction can be gauged by the tabulated values of $S_{2 \pi}^{B O}$ which are all very high, indicating that the reaction intrinsically involves both adiabatic states not just a single one. The entanglement $S_{2 \pi}^{L}$ between the reactant and product states that could be used in a quantum information processor with spatial detectors remains very low, however [122], indicating the unsuitability of processes in this regime for quantum computing applications.

Trajectories (m)-(p) are for scenarios in the delocalized limit (Fig. 1) with $2 J / \lambda=1.5$. All four cases lead to intense electronic spectra (Fig. 2). A component coming from vibrational transitions is also found that grows in relative strength as the reaction becomes more endothermic, perhaps reaching a maximum at around $E_{0} / \lambda=0.25$ (case $(\mathrm{m})$ ) owing to increasing initial wavepacket displacement covering an energy range in which the ground-state surface is highly anharmonic. The symmetric system (n) shows a single line in the electronic spectrum representing the origin of the electronic transition, with a weak additional infrared transition coming from the mismatch of the wavepacket with the significantly anharmonic ground-state potential. This situation mimics the standard spectroscopy of aromatic molecules. Of course Franck-Condon effects associated with motions other than the reaction coordinate broaden this line into a band when multiple nuclear motions are included in calculations.

Trajectories (e)-(h) are for typical reactions over transition states and have $2 J / \lambda=0.3$, whilst (i)-(l) are for more unusual reactions over transition states close to the changeover region to the delocalized regime (Fig. 1). Both sets show smoothly varying properties intermediate between those of the weak-coupling ((a)-(d)) and delocalized ((m)-(p)) scenarios. For (e), tunneling spectra continue to dominate as in (a), but the anharmonicity introduced by the stronger electronic coupling facilitates vibrational transitions also, giving rise to a distorted band. Electronic transitions become important only for the related stronger coupling scenario, (h). As these reaction become more exothermic, the electronic absorption component grows to become dominant, while the tunneling transitions disappear to leave only simple vibrational transitions. The most irregular spectra are produced by $(\mathrm{h})$, this being the only case in which interference between vibrational and electronic transitions is pronounced.

The calculated spectra therefore show a wide range of features that could be observed in transition-state spectroscopy, linking smoothly to commonly observed tunneling transitions, 
infrared transitions, and electronic spectroscopic transitions in appropriate limits. Figure 5 shows the time-domain functions $c(t)$ (Eqns. (28)-(29) associated with the total spectra from Fig. 2. These also show a very wide range of responses, both regular periodic-looking motion and rather irregular chaotic-looking motion. In these simple calculations in which only one nuclear motion is included, quantum coherence of the dynamics is assured. The consequences of this coherence become apparent through looking at the irregular features of the transition-state spectra.

In calculations on complex molecules, the Born-Oppenheimer description is often used to calculate spectral and chemical properties. Fig. 3 shows results obtained using this (BO) (Eqn. (5)), the Born-Huang approximation (BH) (Eqn. (20)), and the first-derivative approximation (FD) typically used as part of surface hopping approaches

$$
\mathbf{H} \approx\left[\begin{array}{cc}
T+\varepsilon_{-}(Q) & \Delta P^{F D}(Q) \frac{\partial}{\partial Q} \\
\Delta P^{F D}(Q) \frac{\partial}{\partial Q} & T+\varepsilon_{+}(Q)
\end{array}\right],
$$

where they are compared to results from the full calculation (FC) obtained using all three BornOppenheimer correction terms. Significant deviations are found only for systems in the weakcoupling limit with $2 J / \lambda=0.03$, with the Born-Oppenheimer approximation performing very well for $2 J / \lambda=0.3$ and above. This is because only in the weak-coupling limit does the Born-Huang spike height $\Delta \Delta E^{\dagger}$ become appreciable (see Table 1, and Fig 3). Results performed using the adiabatic Born-Huang approximation always outperform those obtained using standard nonadiabatic surface-hopping approaches. This result is typical of those obtained for many properties comparing the $\mathrm{BH}$ and FD approaches [69], an exception being that only the FD method can manifest entanglement between the Born-Oppenheimer states and so would become advantageous for some observed property that is directly dependent on entanglement [70]. Mostly the results shown in Fig. 4 indicate that the $\mathrm{BH}$ approximation does qualitatively describe transition state spectra, and that many processes considered to be "non-adiabatic" using the Born-Oppenheimer description are indeed "adiabatic" when using the Born-Huang one. Nevertheless, accurate calculations performed to investigate transition-state spectroscopy in the weak-coupling limit should always be performed in a diabatic basis or else must include all three Born-Oppenheimer corrections.

3e. Results for trajectories starting at the transition state.

We also consider the alternate set of trajectories commencing near the transition state, as would be produced following fluorescence from the excited-state potential minimum down to the ground state. Spectra analogous to Fig. 2 are shown in Fig. 6, with the trajectories obtained using the full calculation in the Born-Oppenheimer basis shown in Fig. 7. Again a wide mix of vibrational and electronic transitions can be produced, with the trends in Fig. (6) having many parallels to those in Fig. (2). For all cases except those with $2 J / \lambda=0.03$ in the weak-coupling limit, the electronic spectrum contains only a single peak corresponding to the opposite process to the emission that created the initial wavepacket. In the weak coupling case, the electronic absorption becomes spread out and too weak to discern amongst the vibrational transitions on the ground state. These transitions grow rapidly in intensity, becoming more strongly allowed than the reverse electronic absorption. The vibrational transitions associated with the transition-state 
spectroscopy are ordered in the inverted region but chaotic otherwise. It is clear that all spectra contain much detailed information relating to the system properties.

\section{Conclusions}

Within the scope of the basic one-dimensional generalized adiabatic electron-transfer theory model, a wide variety of transition-state spectra and dynamics can be produced. The model reveals how transition-state spectroscopy reduces to standard molecular spectroscopy in the strong-coupling delocalized regime, and how it can lead to very complex results for both typical transition states as well as for processes in the non-adiabatic and inverted regimes. Vibrational absorption and emission spectra may be very weak, but, throughout the parameter space of the model, they contain important information usable to interpret system properties. Approximate treatments based on surface hopping are found always to give poor descriptions of dynamics and spectra evaluated using full quantum dynamics, with inclusion in approximate methods of only the typically neglected diagonal term without any non-adiabatic coupling typically performing much better than surface hoping approaches that ignore it.

\section{References}

[1] M. Dantus, M.J. Rosker, A.H. Zewail, Real-time femtosecond probing of "transition states" in chemical reactions, J. Chem. Phys. 87 (1987) 2395-2397.

[2] M. Dantus, M.J. Rosker, A.H. Zewail, Femtosecond real-time probing of reactions. II. The dissociation reaction of ICN, J. Chem. Phys. 89 (1988) 6128-6140.

[3] M.J. Rosker, M. Dantus, A.H. Zewail, Femtosecond real-time probing of reactions. I. The technique, J. Chem. Phys. 89 (1988) 6113-6127.

[4] J.C. Polanyi, A.H. Zewail, Direct Observation of the Transition State, Acc. Chem. Res. 28 (1995) 119-132.

[5] R.M. Bowman, M. Dantus, A.H. Zewail, Femtochemistry of the reaction: IHgI* $\rightarrow$ [IHg...I] t $^{*} \rightarrow$ HgI+I, Chem. Phys. Lett. 156 (1989) 131-137.

[6] A.H. Zewail, Femtochemistry: Recent progress in studies of dynamics and control of reactions and their transition states, J. Phys. Chem. 100 (1996) 12701-12724.

[7] G. He, Femtochemistry - A dark horse?, Progress in Chemistry 9 (1997) X4-150.

[8] A.H. Zewail, Femtochemistry: Atomic-scale dynamics of the chemical bond using ultrafast lasers (Nobel lecture), Angewandte Chemie - International Edition 39 (2000) 2586-2631.

[9] O.M. Sarkisov, S.Y. Umanskii, Femtochemistry, Usp. Khim. 70 (2001) 536-538.

[10] H. Petek, S. Ogawa, Surface femtochemistry: Observation and quantum control of frustrated desorption of alkali atoms from noble metals, Annu. Rev. Phys. Chem., 2002, pp. 507-531.

[11] E. Charron, D.T. Stibbe, A. Suzor-Weiner, Femtosecond dynamics of NaI and CsI aggregated with CH3CN, Femtochemistry and Femtobiology: Ultrafast Events in Molecular Science2004, pp. 115-118.

[12] A. Materny, Femtochemistry: Elementary steps of chemical reactions, Chemie in Unserer Zeit 40 (2006) 226-237.

[13] N.E. Henriksen, Femtochemistry - Some reflections and perspectives, Chem. Phys. 442 (2014) 2-8.

[14] V. Engel, H. Metiu, R. Almeida, R.A. Marcus, A.H. Zewail, Molecular state evolution after excitation with an ultra-short laser pulse: A quantum analysis of $\mathrm{NaI}$ and $\mathrm{NaBr}$ dissociation, Chem. Phys. Lett. 152 (1988) 1-7.

[15] E.J. Heller, The semiclassical way to molecular spectroscopy, Acc. Chem. Res. 14 (1981) 368375. 
[16] K.C. Kulander, E.J. Heller, Time dependent formulation of polyatomic photofragmentation: Application to H3+, J. Chem. Phys. 69 (1978) 2439-2449.

[17] J.R. Reimers, K.R. Wilson, E.J. Heller, Complex time dependent wave packet technique for thermal equilibrium systems: Electronic spectra, J. Chem. Phys. 79 (1983) 4749.

[18] G. Wu, P. Hockett, A. Stolow, Time-resolved photoelectron spectroscopy: From wavepackets to observables, Phys. Chem. Chem. Phys. 13 (2011) 18447-18467.

[19] D.J. Tannor, S.A. Rice, Coherent pulse sequence control of product formation in chemical reactions, Adv. Chem. Phys. 70 (1988) 441-523.

[20] K. Hoki, Y. Ohtsuki, H. Kono, Y. Fujimura, Quantum Control of NaI Predissociation in Subpicosecond and Several-Picosecond Time Regimes, J. Phys. Chem. A 103 (1999) 6301-6308.

[21] A. Glaß, T. Rozgonyi, T. Feurer, R. Sauerbrey, G. Szabó, Control of the photodissociation of CsCl, Appl. Phys. B: Lasers Opt. 71 (2000) 267-276.

[22] A. Lindinger, V. Bonačić-Koutecký, R. Mitrić, D. Tannor, C.P. Koch, V. Engel, T.M. Bernhardt, J. Jortner, A. Mirabal, L. Wöste, Analysis and control of small isolated molecular systems, Springer Series in Chemical Physics, 2007, pp. 25-152.

[23] V. Engel, C. Meier, D.J. Tannor, Local control theory: Recent applications to energy and particle transfer processes in molecules, Adv. Chem. Phys., 2009, pp. 29-101.

[24] D. Avisar, D.J. Tannor, Multi-dimensional wavepacket and potential reconstruction by resonant coherent anti-Stokes Raman scattering: Application to H $2 \mathrm{O}$ and HOD, J. Chem. Phys. 136 (2012).

[25] D. Avisar, D.J. Tannor, Excited-state wavepacket and potential reconstruction by coherent anti-Stokes Raman scattering, Phys. Chem. Chem. Phys. 17 (2015) 2297-2310.

[26] M. Shapiro, P. Brumer, Quantum Control of Molecular Processes: Second Edition, Wiley, Weinheim, 2012.

[27] P. Král, M. Shapiro, Complete adiabatic passage to arbitrarily sculpted superposition states, Physical Review A. Atomic, Molecular, and Optical Physics 65 (2002) 434131-434137.

[28] M. Shapiro, Association, dissociation, and the acceleration and suppression of reactions by laser pulses, Adv. Chem. Phys., 2000, pp. 123-191.

[29] J.R. Waldeck, M. Shapiro, R. Bersohn, Theory of transient anisotropy in molecular photodissociation, J. Chem. Phys. 99 (1993) 5924-5935.

[30] S.Y. Lee, W.T. Pollard, R.A. Mathies, Quasi-classical models of transition state absorption or emission, Chem. Phys. Lett. 163 (1989) 11-18.

[31] J.L. Krause, M. Shapiro, An exact coupled-channel study of the transition state spectroscopy of the collinear H+H2 reaction, J. Chem. Phys. 90 (1989) 6401-6408.

[32] M. Shapiro, R. Bersohn, Vibrational energy distribution of the CH3 radical photodissociated from CH3I, J. Chem. Phys. 73 (1980) 3810-3817.

[33] T.I. Sølling, T.S. Kuhlman, A.B. Stephansen, L.B. Klein, K.B. Møller, The non-ergodic nature of internal conversion, ChemPhysChem 15 (2014) 249-259.

[34] J.P. Bergsma, J.R. Reimers, K.R. Wilson, J.T. Hynes, Molecular dynamics of the A+BC

reaction in rare gas solution, J. Chem. Phys. 85 (1986) 5625.

[35] J.P. Bergsma, P.M. Edelsten, B.J. Gertner, K.R. Huber, J.R. Reimers, K.R. Wilson, S.M. Wu, J.T. Hynes, Dynamics of the A+BC reaction in solution, Chem. Phys. Letts. 123 (1986) 394.

[36] I.V. Rubtsov, K. Yoshihara, Vibrational coherence in electron donor-acceptor complexes, J. Phys. Chem. A 103 (1999) 10202-10212.

[37] K. Wynne, R.M. Hochstrasser, Coherence and adiabaticity in ultrafast electron transfer, Adv. Chem. Phys., 1999, pp. 263-309.

[38] D.P. Hoffman, R.A. Mathies, Femtosecond Stimulated Raman Exposes the Role of Vibrational Coherence in Condensed-Phase Photoreactivity, Acc. Chem. Res. 49 (2016) 616-625.

[39] M.A. Nielsen, I.L. Chuang, Quantum Computation and Quantum Information, Cambridge University Press, New York, 2000.

[40] T. Taneichi, T. Kobayashi, Y. Ohtsuki, Y. Fujimura, Control of non-adiabatic photodissociation of sodium iodide using ultrashort pump and control pulses, Chem. Phys. Lett. 231 (1994) 50-54. 
[41] T. Kobayashi, T. Saito, H. Ohtani, Real-time spectroscopy of transition states in bacteriorhodopsin during retinal isomerization, Nature 414 (2001) 531-534.

[42] T. Kobayashi, A. Shirakawa, T. Fuji, Real-time spectroscopy of molecular vibration using sub5-fs pulses, ACS Symp. Ser., 2002, pp. 171-187.

[43] T. Kobayashi, A. Yabushita, Ultrafast real-time molecular structure studied by broadband gated fourier transform: Femtosecond photo-isomerization in bacteriorhodopsin, Optics InfoBase Conference Papers, 2007.

[44] T. Kobayashi, A. Yabushita, T. Saito, H. Ohtani, M. Tsuda, Sub-5-fs real-time spectroscopy of transition states in bacteriorhodopsin during retinal isomerization, Photochem. Photobiol. 83 (2007) 363-368.

[45] T. Kobayashi, A. Yabushita, Fs photo-isomerization in bacteriorhodopsin by few-cycle pulses, J. Lumin. 128 (2008) 1038-1042.

[46] A. Yabushita, T. Kobayashi, Primary conformation change in bacteriorhodopsin on photoexcitation, Biophys. J. 96 (2009) 1447-1461.

[47] A. Yabushita, T. Kobayashi, Primary events in the photodissociation of oxyhemoglobin, Spectroscopy 24 (2010) 333-338.

[48] A. Yabushita, T. Kobayashi, Vibrational fine structures revealed by the frequency-to-time fourier transform of the transient spectrum in Bacteriorhodopsin, J. Phys. Chem. B 114 (2010) $4632-4636$.

[49] T. Kobayashi, A. Yabushita, Transition-state spectroscopy using ultrashort laser pulses, Chem. Rec. 11 (2011) 99-116.

[50] T. Kobayashi, Development of ultrafast spectroscopy and reaction mechanisms studied by the observation of ultrashort-life species and transition states, Bull. Chem. Soc. Jpn. 86 (2013) 167-182. [51] W. Domcke, G. Stock, Theory of ultrafast nonadiabatic excited-state processes and their spectroscopic detection in real time, Adv. Chem. Phys., 1997, pp. 1-169.

[52] B. Wolfseder, L. Seidner, G. Stock, W. Domcke, Femtosecond pump-probe spectroscopy of electron-transfer systems: A nonperturbative approach, Chem. Phys. 217 (1997) 275-287.

[53] A.E. Boguslavskiy, M.S. Schuurman, D. Townsend, A. Stolow, Non-Born-Oppenheimer wavepacket dynamics in polyatomic molecules: Vibrations at conical intersections in DABCO, Faraday Discuss. 150 (2011) 419-438.

[54] T. Yonehara, K. Hanasaki, K. Takatsuka, Fundamental approaches to nonadiabaticity: Toward a chemical theory beyond the born-oppenheimer paradigm, Chem. Rev. 112 (2012) 499-542.

[55] K. Yamamoto, K. Takatsuka, Electronic quantum effects mapped onto non-Born-Oppenheimer nuclear paths: Nonclassical surmounting over potential barriers and trapping above the transition states due to nonadiabatic path-branching, J. Chem. Phys. 140 (2014).

[56] H.S. You, S. Han, J.H. Yoon, J.S. Lim, J. Lee, S.Y. Kim, D.S. Ahn, J.S. Lim, S.K. Kim, Structure and dynamic role of conical intersections in the $\pi \sigma^{*}$-mediated photodissociation reactions, Int. Rev. Phys. Chem. 34 (2015) 429-459.

[57] R.A. Marcus, N. Sutin, Electron transfers in chemistry and biology, Biochim. Biophys. Acta 811 (1985) 265-322.

[58] N.S. Hush, Inequivalent XPS [x-ray photoelectron spectroscopy] binding energies in symmetrical delocalized mixed-valence complexes, Chem. Phys. 10 (1975) 361.

[59] N.S. Hush, Electron delocalization, structure and dynamics in mixed-valence systems, NATO Adv. Study Inst. Ser., Ser. C 58 (1980) 151-188.

[60] N.S. Hush, Quantum-mechanical discussion of the gas phase formation of quinonedimethide monomers, J. Polymer Sci. 11 (1953) 289-298.

[61] N.S. Hush, Adiabatic rate processes at electrodes, J. Chem. Phys. 28 (1958) 962-972.

[62] N.S. Hush, in: A.N. Frumkin (Ed.), Soviet electrochemistry: Proceedings of the Fourth Conference on Electrochemistry 1956, Consultants Bureau, New York, 1961, pp. 99-100.

[63] N.S. Hush, Adiabatic theory of outer-sphere electron-transfer processes in solution, Trans.

Farad. Soc. 57 (1961) 577-580.

[64] N.S. Hush, Intervalence-transfer absorption. II. Theoretical considerations and spectroscopic data, Prog. Inorg. Chem. 8 (1967) 391-444. 
[65] N.S. Hush, Electron transfer in retrospect and prospect 1: adiabatic electrode processes, J. Electroanal. Chem. 460 (1999) 5.

[66] P. Day, N.S. Hush, R.J.H. Clark, Mixed valence: origins and developments, Phil. Trans. Roy. Soc. A 366 (2008) 5-14.

[67] J.R. Reimers, L. McKemmish, R.H. McKenzie, N.S. Hush, A unified diabatic description for electron transfer reactions, isomerization reactions, proton transfer reactions, and aromaticity, Phys. Chem. Chem. Phys. 17 (2015) 24598-25617.

[68] J.R. Reimers, L. McKemmish, R.H. McKenzie, N.S. Hush, Bond angle variations in XH3 [X=N,P,As, Sb,Bi]: the critical role of Rydberg orbitals exposed using a diabatic state model, Phys. Chem. Chem. Phys. 17 (2015) 24618-24640.

[69] J.R. Reimers, L. McKemmish, R.H. McKenzie, N.S. Hush, Non-adiabatic effects in thermochemistry, spectroscopy and kinetics: the general importance of all three Born-Oppenheimer breakdown corrections, Phys. Chem. Chem. Phys. 17 (2015) 24640-24665

[70] L. McKemmish, R.H. McKenzie, N.S. Hush, J.R. Reimers, Electron-vibration entanglement in the Born-Oppenheimer description of chemical reactions and spectroscopy, Phys. Chem. Chem. Phys. 17 (2015) 24666-24682.

[71] L.K. McKemmish, D.J. Kedziora, G.R. White, N.S. Hush, J.R. Reimers, Frequency-based Quantum Computers from a Chemist's Perspective, Aust. J. Chem. 65 (2012) 512-519.

[72] S. Scheit, Y. Arasaki, K. Takatsuka, Controlled dynamics at an avoided crossing interpreted in terms of dynamically fluctuating potential energy curves, J. Phys. Chem. A 116 (2012) 2644-2653. [73] J.R. Reimers, The importance of motions that accompany those occurring along the reaction coordinate, Aust. J. Chem. 68 (2015) 1202-1212.

[74] O. Tishchenko, D.G. Truhlar, A. Ceulemans, M.T. Nguyen, A Unified Perspective on the Hydrogen Atom Transfer and Proton-Coupled Electron Transfer Mechanisms in Terms of Topographic Features of the Ground and Excited Potential Energy Surfaces As Exemplified by the Reaction between Phenol and Radicals, J. Am. Chem. Soc. 130 (2008) 7000-7010.

[75] Y.J. Yan, S. Mukamel, Electronic dephasing, vibrational relaxation, and solvent friction in molecular nonlinear optical line shapes, J. Chem. Phys. 89 (1988) 5160-5176.

[76] T. Shida, The chemical bond: A fundamental quantum-mechanical picture, Springer Series in Chemical Physics, 2004, pp. iii-201.

[77] B.I. Grimberg, V.V. Lozovoy, M. Dantus, S. Mukamel, Ultrafast nonlinear spectroscopic techniques in the gas phase and their density matrix representation, J. Phys. Chem. A 106 (2002) 697-718.

[78] F. London, Zur Quantentheorie der homöopolaren Valenzzahlen, Z. Phys. 46 (1928) 455-477.

[79] F. London, On the theory of non-adiabatic chemical reactions, Z. Phys. 74 (1932) 143.

[80] H. Eyring, M. Polanyi, Concerning simple gas reactions, Z. Phys. Chem. Abt. B 12 (1931) 279-311.

[81] M.G. Evans, M. Polanyi, Inertia and driving force of chemical reactions, Trans. Faraday Soc. 34 (1938) 11-24.

[82] J. Horiuti, M. Polanyi, Outlines of a theory of proton transfer, J. Molec. Catalysis A 199 (2003) 185-197.

[83] F.T. Wall, G. Glockler, The Double Minimum Problem Applied to the Ammonia Molecules, J. Chem. Phys. 5 (1937) 314.

[84] N.S. Hush, Quantum-mechanical discussion of the gas-phase formation of quinonedimethide monomers, J. Polym. Sci. 11 (1953) 289-298.

[85] S.B. Piepho, E.R. Krausz, P.N. Schatz, Vibronic coupling model for calculation of mixed valence absorption profiles, J. Am. Chem. Soc. 100 (1978) 2996-3005.

[86] J.R. Reimers, N.S. Hush, Hamiltonian operators including both symmetric and antisymmetric vibrational modes for vibronic-coupling and intervalence charge-transfer applications, Chem. Phys. 299 (2004) 79.

[87] E.B. Wilson, J.C. Decius, P.C. Cross, Molecular Vibrations: The Theory of Infrared and Raman Vibrational Spectra, McGraw-Hill Book Company, New York, 1955. 
[88] I.B. Bersuker, Pseudo-Jahn-Teller Effect - A Two-State Paradigm in Formation, Deformation, and Transformation of Molecular Systems and Solids, Chem. Rev. 113 (2013) 1351-1390.

[89] S. Zilberg, Y. Haas, D. Danovich, S. Shaik, The twin-excited state as a probe for the transition state in concerted unimolecular reactions: the semibullvalene rearrangement, Angew. Chem., Int. Ed. 37 (1998) 1394-1397.

[90] S. Shaik, S. Zilberg, Y. Haas, A Kekulé-Crossing Model for the “Anomalous” Behavior of the b2u Modes of Aromatic Hydrocarbons in the Lowest Excited 1B2u State, Acc. Chem. Res. 29 (1996) 211-218.

[91] S. Shaik, A. Shurki, D. Danovich, P.C. Hiberty, Origins of the Exalted b2u Frequency in the First Excited State of Benzene, J. Am. Chem. Soc. 118 (1996) 666-671.

[92] S. Shaik, A. Shurki, D. Danovich, P.C. Hiberty, A Different Story of $\pi$-Delocalization-The Distortivity of $\pi$-Electrons and Its Chemical Manifestations, Chem. Rev. (Washington, D. C.) 101 (2001) 1501-1539.

[93] S. Zilberg, Y. Haas, Frequency Upshift in BO2 and CO2+ upon Electronic Excitation: A TwinState Model Rationalization, J. Phys. Chem. A 115 (2011) 10650-10654.

[94] M. Born, K. Huang, Dynamical Theory of Crystal Lattices, Clarendon, Oxford, 1954.

[95] C.J. Ballhausen, A.E. Hansen, Electronic Spectra, Annu. Rev. Phys. Chem. 23 (1972) 15-38.

[96] M. Born, R. Oppenheimer, Zur Quantentheorie der Molekeln, Ann. Phys. 84 (1927) 457-484.

[97] F. London, Ueber den Mechanismus der homöopolaren Bindung. , in: A. Sommerfeld, P.

Debye (Eds.), Probleme der Modernen Physik, Hirsel, Leipzig, 1928, pp. 104-113.

[98] P.T. Saunders, An introduction to catastrophe theory, Cambridge University Press, Cambridge, 1980.

[99] F. Xu, Application of catastrophe theory to the $\Delta \mathrm{G}^{-}$to $-\Delta \mathrm{G}$ relationship in electron transfer reactions, Z. Phys. Chem. 166 (1990) 79-91.

[100] X. Krokidis, B. Silvi, C. Dezarnaud-Dandine, A. Sevin, Topological study, using a coupled ELF and catastrophe theory technique, of electron transfer in the $\mathrm{Li}+\mathrm{Cl} 2$ system, New J. Chem. 22 (1998) 1341-1350.

[101] D.J. Wales, A Microscopic Basis for the Global Appearance of Energy Landscapes, Science 293 (2001) 2067-2070.

[102] A. Toro-Labbe, Characterization of Chemical Reactions from the Profiles of Energy, Chemical Potential, and Hardness, J. Phys. Chem. A 103 (1999) 4398-4403.

[103] A. Toro-Labbe, S. Gutierrez-Oliva, J.S. Murray, P. Politzer, A new perspective on chemical and physical processes: the reaction force, Mol. Phys. 105 (2007) 2619-2625.

[104] A. Toro-Labbe, S. Gutierrez-Oliva, J.S. Murray, P. Politzer, The reaction force and the transition region of a reaction, J. Mol. Model. 15 (2009) 707-710.

[105] P. Politzer, J.R. Reimers, J.S. Murray, A. Toro-Labbe, Reaction Force and Its Link to Diabatic Analysis: A Unifying Approach to Analyzing Chemical Reactions, J. Phys. Chem. Lett. 1 (2010) 2858-2862.

[106] R.A. Marcus, Chemical and electrochemical electron-transfer theory, Annu. Rev. Phys. Chem. 15 (1964) 155-196.

[107] R. Kubo, Y. Toyozawa, Application of the Method of Generating Function to Radiative and Non-Radiative Transitions of a Trapped Electron in a Crystal, Prog. Theor. Phys. 13 (1955) 160. [108] U. Öpik, M.H.L. Pryce, Studies of the Jahn-Teller Effect. I. A Survey of the Static Problem, Proc. R. Soc. London, A 238 (1957) 425-447.

[109] V.G. Levich, R.R. Dogonadze, Theory of rediationless electron transitions between ions in solution, Dokl. Akad. Nauk. SSSR Ser. Fiz. Khim. 124 (1959) 123-126.

[110] V.G. Levich, R.R. Dogonadze, Adiabatic theory for electron-transfer processes in solution, Dokl. Akad. Nauk. SSSR Ser. Fiz. Khim. 133 (1960) 158-161.

[111] V.G. Levich, R.R. Dogonadze, Adiabatic theory of electron-transfer processes in solution, Collect. Czech. Chem. Commun. 26 (1961) 193-214.

[112] V.G. Levich, Present state of the theory of oxidation=reduction in solution (bulk and electrode reactions), in: P. Delahaye (Ed.), Advances in Electrochemistry and Electrochemical Engineering Vol. 4, Interscience Publishers, New York, 1966, pp. 249-371. 
[113] J.C. Tully, Nonadiabatic dynamics, Modern Methods for Multidimensional Dynamics Computations in Chemistry (1998).

[114] J.C. Tully, Perspective: Nonadiabatic dynamics theory, J. Chem. Phys. 137 (2012) 22 A301.

[115] J.R. Reimers, N.S. Hush, The effects of couplings to symmetric and antisymmetric modes and minor asymmetries on the spectral properties of mixed-valence and related charge-transfer systems, Chem. Phys. 208 (1996) 177.

[116] J.R. Reimers, N.S. Hush, Electric field perturbation of electronic (vibronic) absorption envelopes: Application to characterization of mixed-valence states, in: K. Prassides (Ed.), Mixed Valence Systems: Applications in Chemistry, Physics, and Biology, Kluwer Acad. Publishers, Dordrecht, 1991, pp. 29-50.

[117] J.R. Reimers, N.S. Hush, Electronic properties of transition-metal complexes determined from electro-absorption spectroscopy II. mono-nuclear complexes of ruthenium(II), J. Phys. Chem. 95 (1991) 9773.

[118] T.A. Costi, R.H. McKenzie, Entanglement between a qubit and the environment in the spinboson model, Phys. Rev. A 68 (2003) 034301/034301-034301/034304.

[119] S.Y. Cho, R.H. McKenzie, Quantum entanglement in the two-impurity Kondo model, Phys. Rev. A 73 (2006) 012109.

[120] A.J. Ferguson, P.A. Cain, D.A. Williams, G.A.D. Briggs, Ammonia-based quantum computer, Phys. Rev. A 65 (2002) 034303.

[121] J.R. Reimers, N.S. Hush, Electron and energy transfer through bridged systems. I. Formalism, Chem. Phys. 134 (1989) 323.

[122] L.K. McKemmish, R.H. McKenzie, N.S. Hush, J.R. Reimers, Quantum entanglement between electronic and vibrational degrees of freedom in molecules, J. Chem. Phys. 135 (2011) 244110. 
Table 1. Properties of the 16 wavepacket trajectories starting in the reactant region, see Figs. 1$5^{\mathrm{a}}$

\begin{tabular}{ccccccccccc}
\hline Traj. & $\frac{2|J|}{\lambda}$ & \multirow{2}{*}{$Q_{\mathrm{C}}$} & $\frac{\Delta \Delta E^{\ddagger}}{\hbar \omega}$ & $\frac{E_{0}}{\Delta E}$ & $\frac{\Delta E^{\ddagger} \mathrm{b}}{\hbar \omega}$ & $f_{\mathrm{el}}$ & $\kappa_{2 \pi}$ & $f_{\pi}^{+}$ & $S_{2 \pi}^{\mathrm{CA}}$ & $S_{2 \pi}^{\mathrm{BO}}$ \\
\hline $\mathrm{a}$ & 0.03 & 0.07 & 28 & 0.25 & 3.76 & 0.0001 & 0.03 & 0.43 & 0.07 & 0.75 \\
$\mathrm{~b}$ & & & & 0 & 2.35 & 0.0002 & 0.03 & 0.42 & 0.08 & 0.94 \\
$\mathrm{c}$ & & & & -0.25 & 3.76 & 0.0004 & 0.04 & 0.40 & 0.10 & 0.97 \\
$\mathrm{~d}$ & & & & -1.1 & {$[0.03]$} & 0.10 & 0.52 & 0.47 & 1.00 & 0.99 \\
\hline $\mathrm{e}$ & 0.3 & 0.66 & 0.29 & 0.25 & 2.62 & 0.016 & 0.52 & 0.05 & 0.81 & 0.28 \\
$\mathrm{f}$ & & & & 0 & 1.17 & 0.030 & 0.56 & 0.03 & 0.83 & 0.18 \\
$\mathrm{~g}$ & & & & -0.25 & 2.62 & 0.079 & 0.68 & 0.02 & 0.87 & 0.11 \\
$\mathrm{~h}$ & & & & -1.1 & {$[0.05]$} & 0.61 & 0.84 & 0.05 & 0.28 & 0.30 \\
\hline $\mathrm{i}$ & 0.8 & \multirow{2}{*}{1.58} & 0.05 & 0.25 & {$[3.40]$} & 0.13 & 0.42 & 0.002 & 0.92 & 0.014 \\
$\mathrm{j}$ & & & & 0 & 0.08 & 0.36 & 0.75 & 0.001 & 0.89 & 0.013 \\
$\mathrm{k}$ & & & & -0.25 & {$[0.90]$} & 0.76 & 0.84 & 0.001 & 0.62 & 0.006 \\
$\mathrm{l}$ & & & & -1.1 & {$[0.33]$} & 0.85 & 0.69 & 0.001 & 0.07 & 0.011 \\
\hline $\mathrm{m}$ & 1.5 & 2.50 & 0.02 & 0.25 & {$[2.92]$} & 0.35 & 0.28 & 0.0004 & 0.88 & 0.005 \\
$\mathrm{n}$ & & & & 0 & {$[1.38]$} & 0.82 & 0.50 & 0.0000 & 0.27 & 0.001 \\
$\mathrm{o}$ & & & & -0.25 & {$[0.42]$} & 0.88 & 0.71 & 0.0002 & 0.55 & 0.002 \\
$\mathrm{p}$ & & & & -1.1 & {$[1.34]$} & 0.93 & 0.60 & 0.0003 & 0.12 & 0.004 \\
\hline
\end{tabular}

a: all at $\hbar \omega / \Delta E=0.1$.

b: the activation energy if a Born-Oppenheimer transition-state exists, else [] the relative energy of the crossover point from Eqn. (16). 


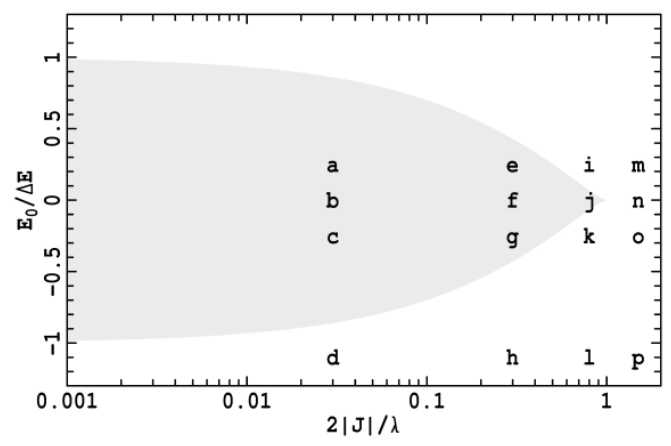

Fig. 1. The shaded region indicates when the ground-state Born-Oppenheimer potential-energy surface from generalized adiabatic electron-transfer theory displays a double-well potential with a transition state. Marked (a)-(p) are the parameter values used in the 16 quantum wavepacket trajectories and spectral simulations used in Figs. 2-7 and Table 1.

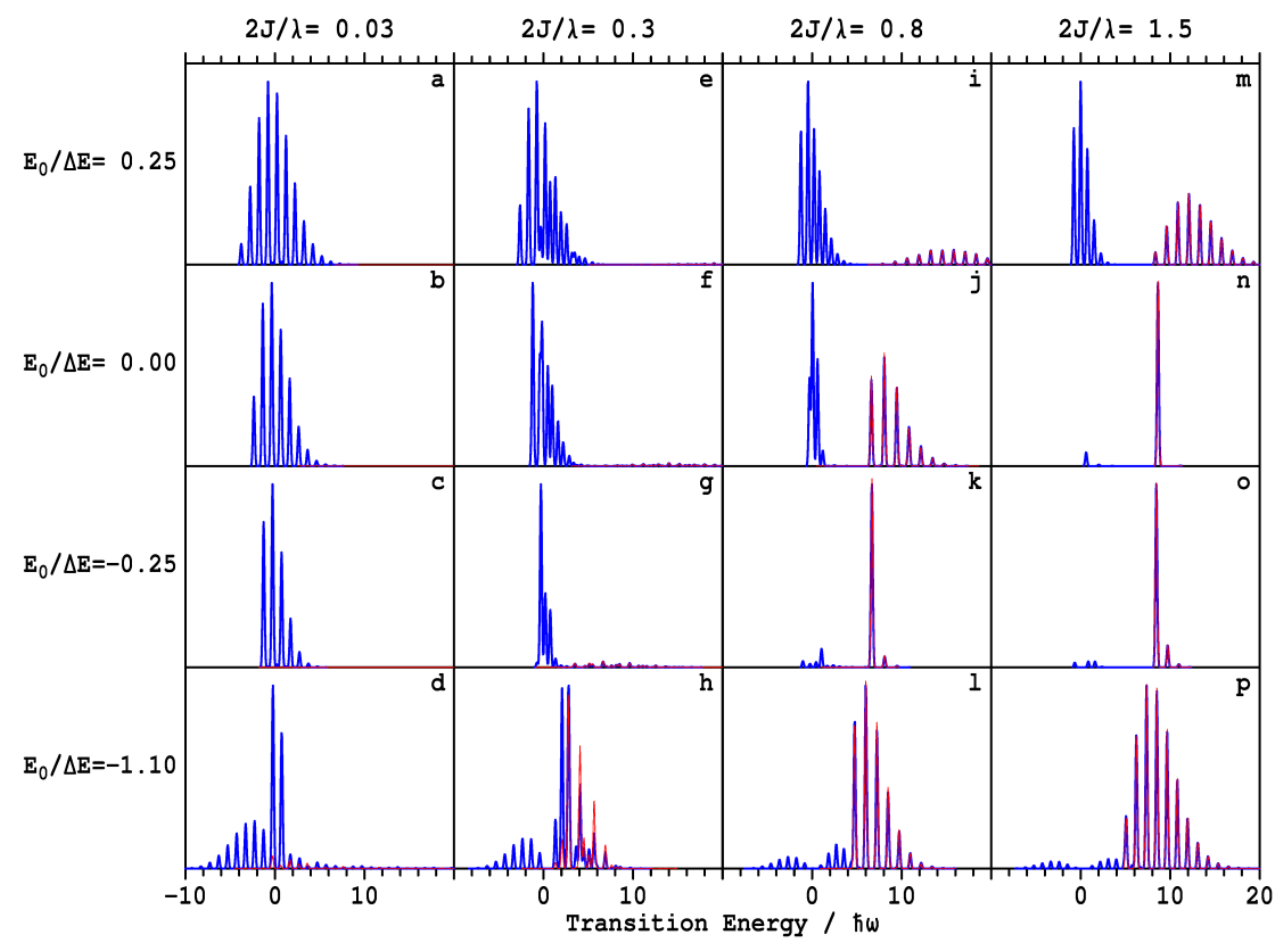

Fig. 2. Spectral profiles for 16 wavepackets (a)-(p) starting on the reactant side at the energy of the transition state (if this exists, at the energy of the lower adiabatic surface at the crossover geometry $Q_{x}$ otherwise) from generalized adiabatic electron-transfer theory. These needs to be scaled by the transition frequency to obtain adsorption intensities (positive transition energies) or by the frequency cubed to obtain emission intensities (negative transition energies. Shown in blue are total spectral profiles and overlayed in red are their components associated with only the electronic transitions between Born-Oppenheimer surfaces. 


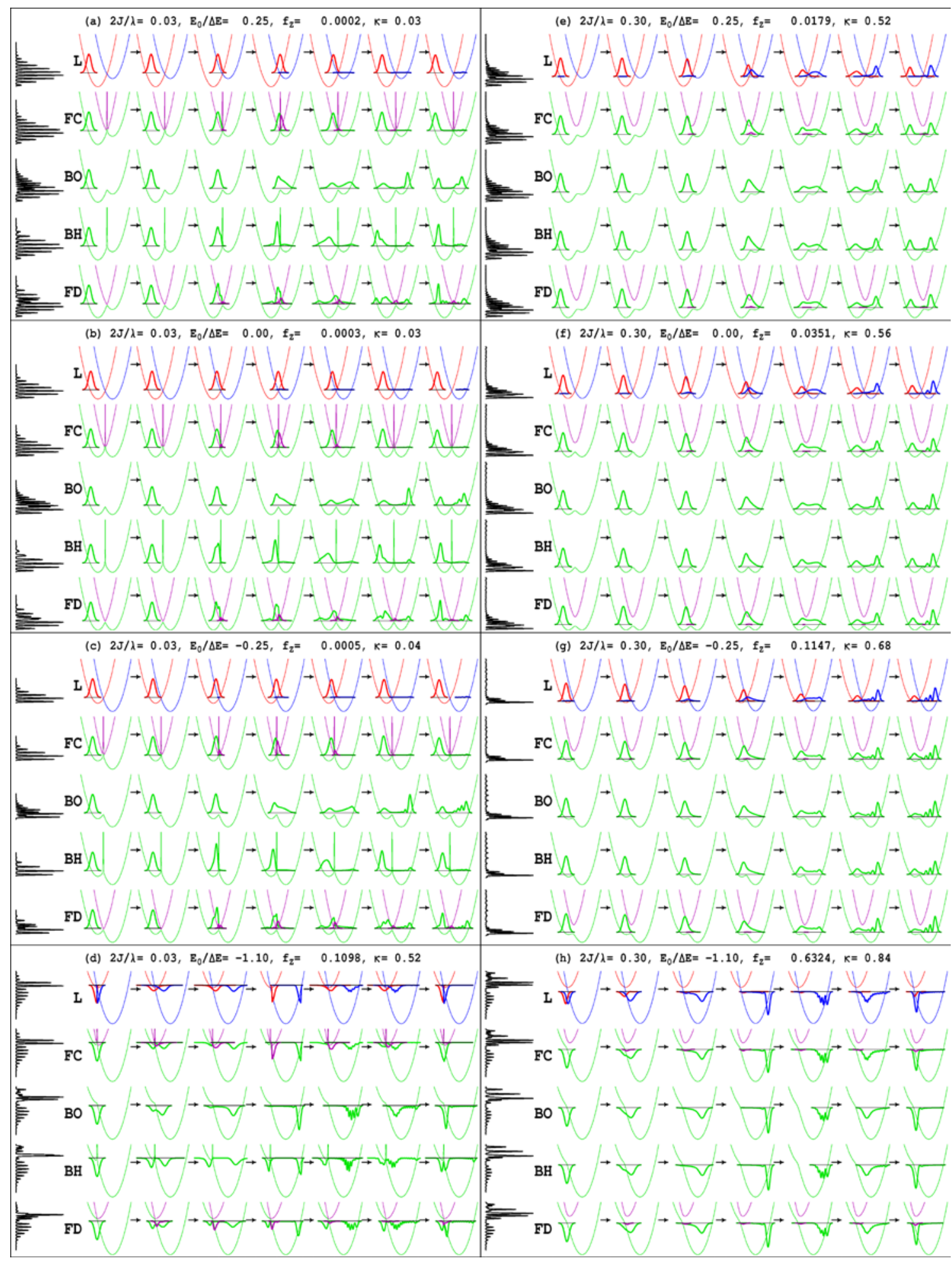

Fig. 3. Wavepacket dynamics and spectra for scenarios (a)-(h), see Fig. 1 and Table 1, starting in the reaction region. The reactant (red) and product (blue) diabatic surfaces are shown, as well as the lower (green) and, if appropriate, upper (magenta) Born-Oppenheimer or Born-Huang adiabatic surfaces. Arrows indicate wavepacket propagation from $t=0 \rightarrow t=\pi / 3 \omega \rightarrow t=2 \pi / 3 \omega \rightarrow t=\pi / \omega \rightarrow t=4 \pi / 3 \omega \rightarrow t=5 \pi / 3 \omega \rightarrow t=2 \pi / \omega$, with wavepacket components coloured for each surface used in its description. The calculation methods used are: L- localized diabatic (crude adiabatic) basis states; FC- full calculation using Born-Oppenheimer basis states; BO- Born-Oppenheimer approximation, BH- BornHuang approximation; FD- surface-hopping approximation. Resulting spectra are shown to the left of the trajectories, with those for CA and FC being equivalent and are shown over a wider frequency range in Fig. 2. 


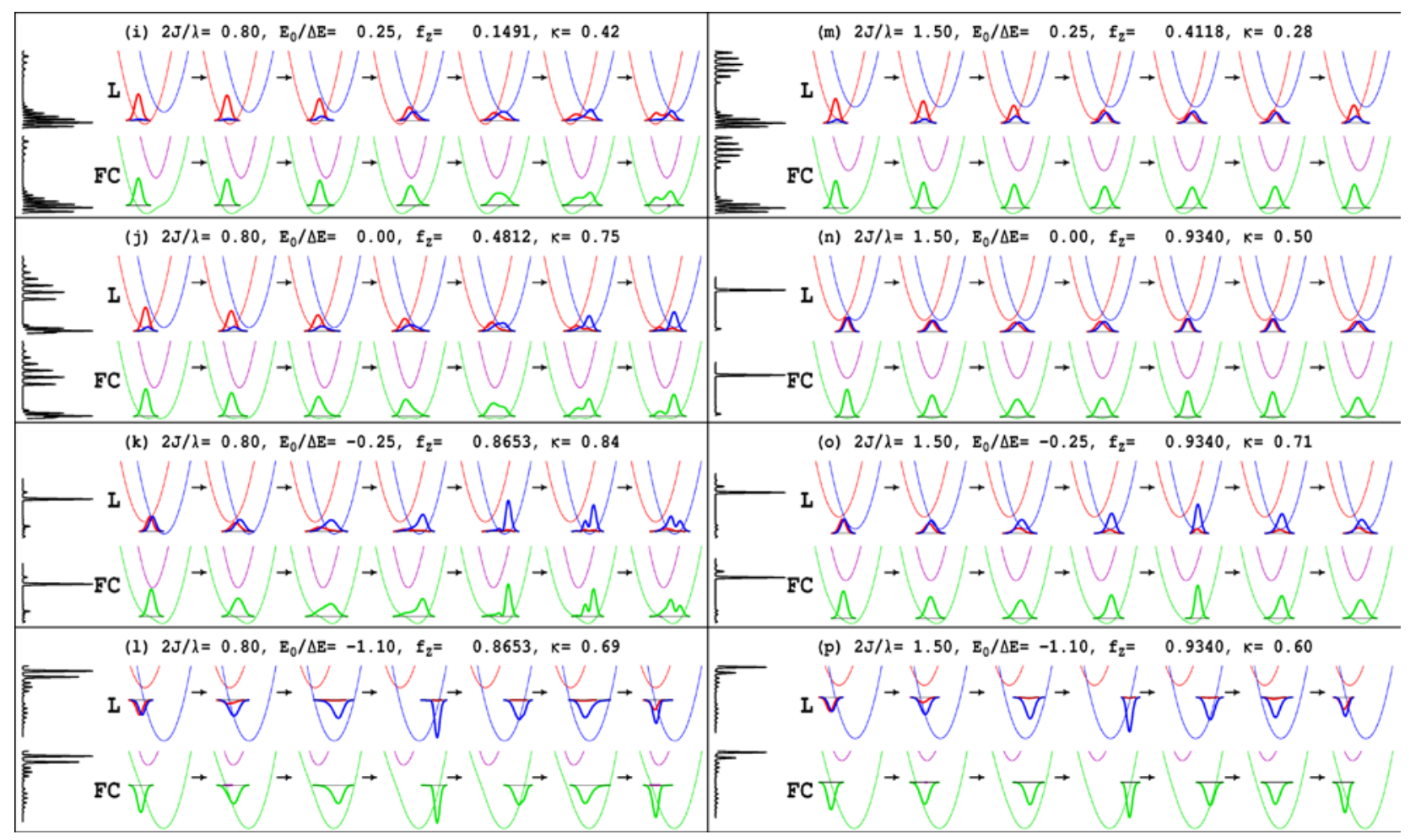

Fig. 4. Wavepacket dynamics and spectra for scenarios (i)-(p), see Fig. 1 and Table 1, starting in the reaction region. The reactant (red) and product (blue) diabatic surfaces are shown, as well as the lower (green) and, if appropriate, upper (magenta) Born-Oppenheimer or Born-Huang adiabatic surfaces. Arrows indicate wavepacket propagation from $t=0 \rightarrow t=\pi / 3 \omega \rightarrow t=2 \pi / 3 \omega \rightarrow t=\pi / \omega \rightarrow t=4 \pi / 3 \omega \rightarrow t=5 \pi / 3 \omega \rightarrow t=2 \pi / \omega$, with wavepacket components coloured for each surface used in its description. L- localized diabatic (crude adiabatic) basis states; FC- full calculation using Born-Oppenheimer basis states. Resulting spectra are shown to the left of the trajectories, with those for CA and FC being equivalent and are shown over a wider frequency range in Fig. 2. 


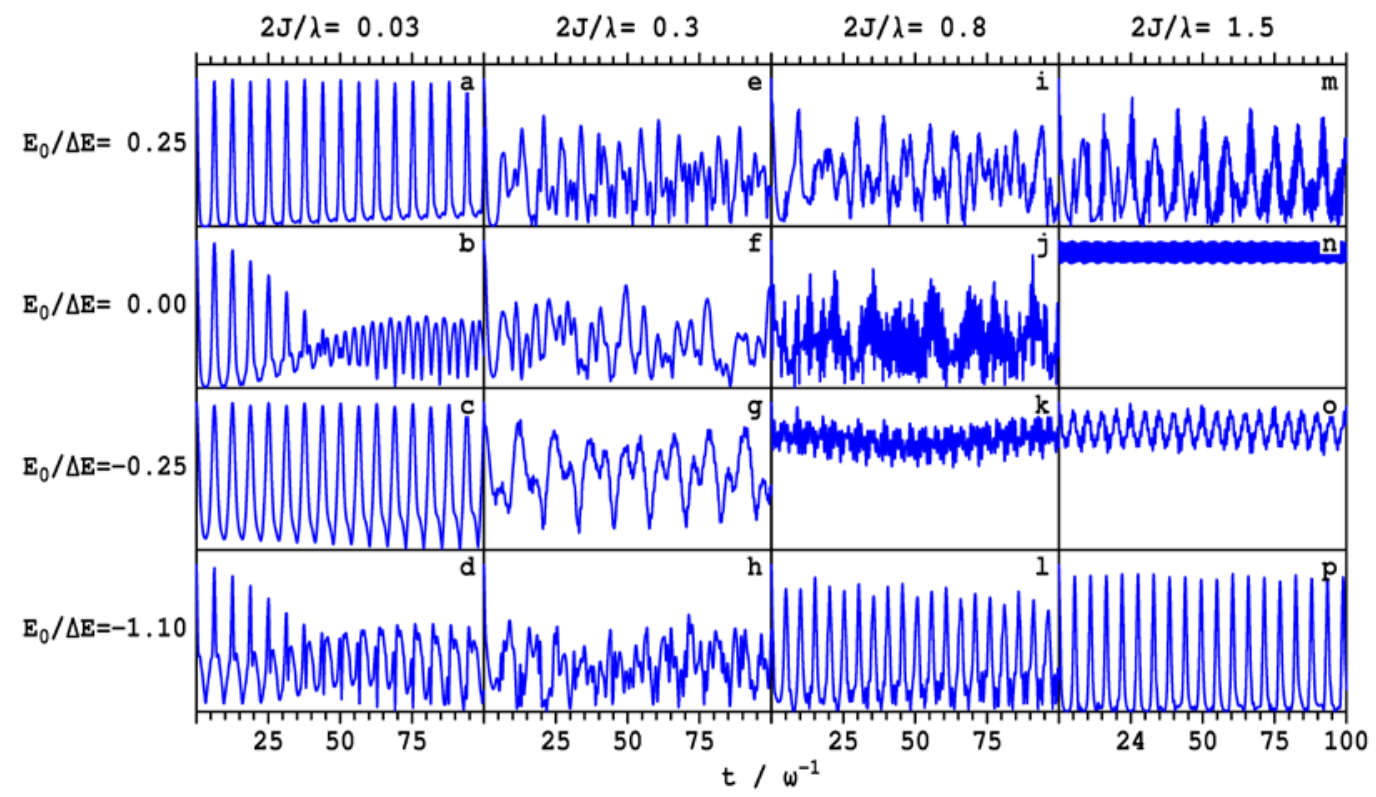

Fig. 5. Autocorrelation functions $c(t)$ associated with the total spectra shown in Fig. 2, Eqn. (28), for wavepackets (a)-(p) starting on the reactant side. 


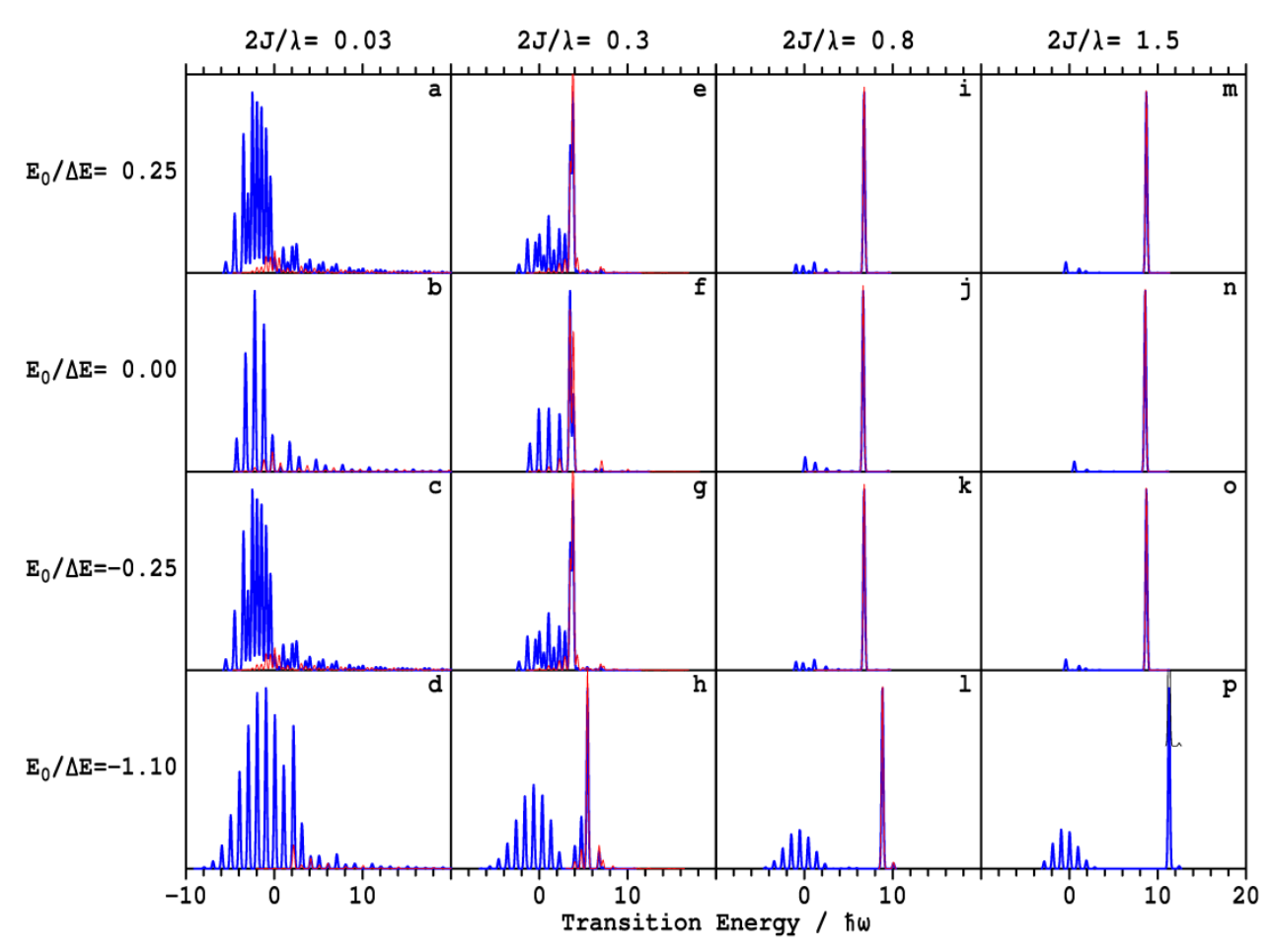

Fig. 6. Spectral profiles for 16 wavepackets (a)-(p) starting at the transition state (if this exists, at the energy of the lower adiabatic surface at the crossover geometry $Q_{x}$ otherwise) from generalized adiabatic electron-transfer theory. These needs to be scaled by the transition frequency to obtain adsorption intensities (positive transition energies) or by the frequency cubed to obtain emission intensities (negative transition energies). Shown in blue are total spectral profiles and overlayed in red are their components associated with only the electronic transitions between Born-Oppenheimer surfaces. 


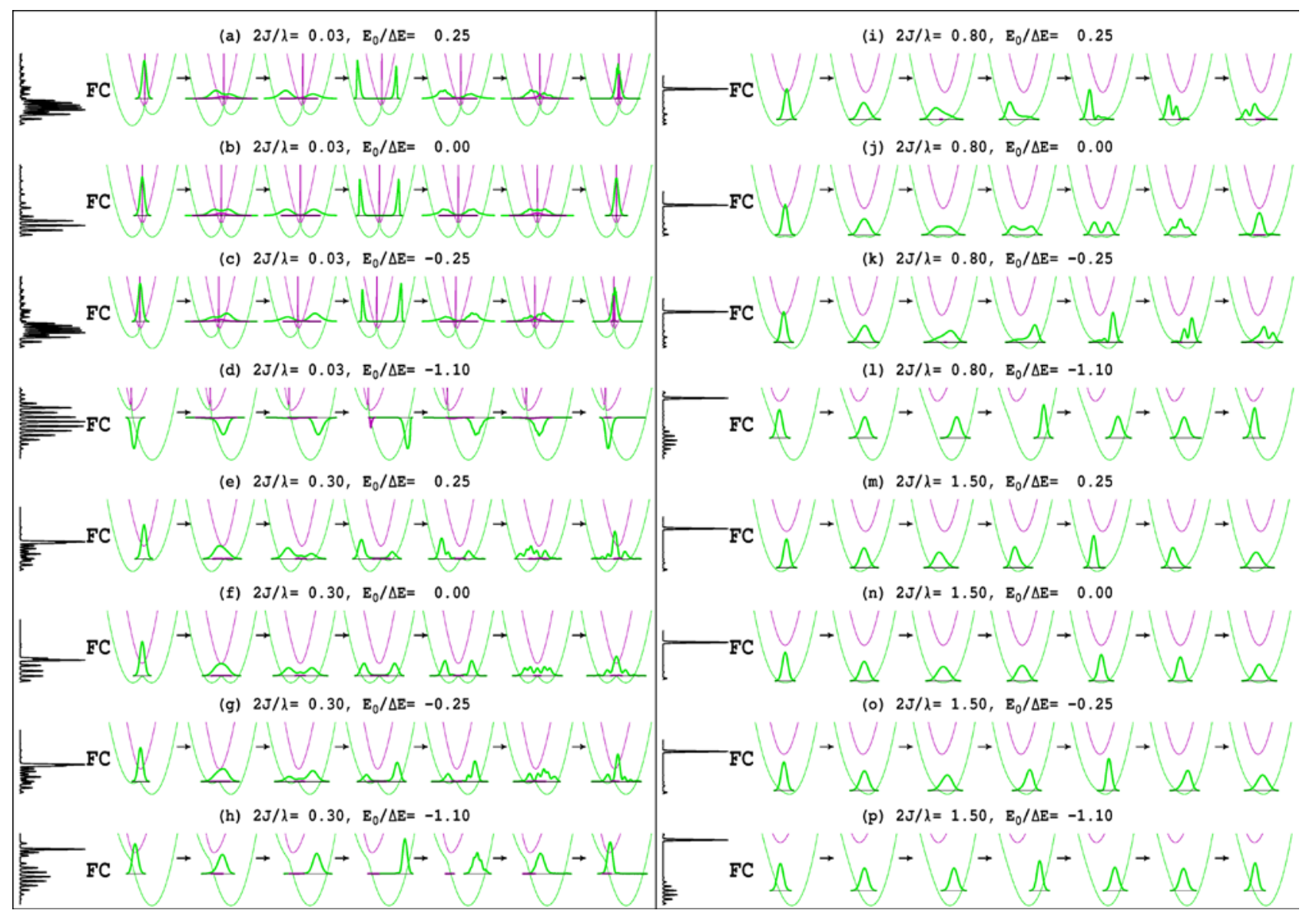

Fig. 7. Wavepacket dynamics and spectra for scenarios (i)-(p), see Fig. 1, starting in the transition-state region. The lower (green) and upper (magenta) Born-Oppenheimer adiabatic surfaces are shown. Arrows indicate wavepacket propagation from $t=0 \rightarrow t=\pi / 3 \omega \rightarrow t=2 \pi / 3 \omega \rightarrow t=\pi / \omega \rightarrow t=4 \pi / 3 \omega \rightarrow t=5 \pi / 3 \omega \rightarrow t=2 \pi / \omega$ using the full calculation in the Born-Oppenheimer basis states. Resulting spectra are shown to the left of the trajectories and also over a wider frequency range in Fig. 6. 
TOC graphic

$531 \times 1328$ pixels $(h \times w) 5 \times 13 \mathrm{~cm}$

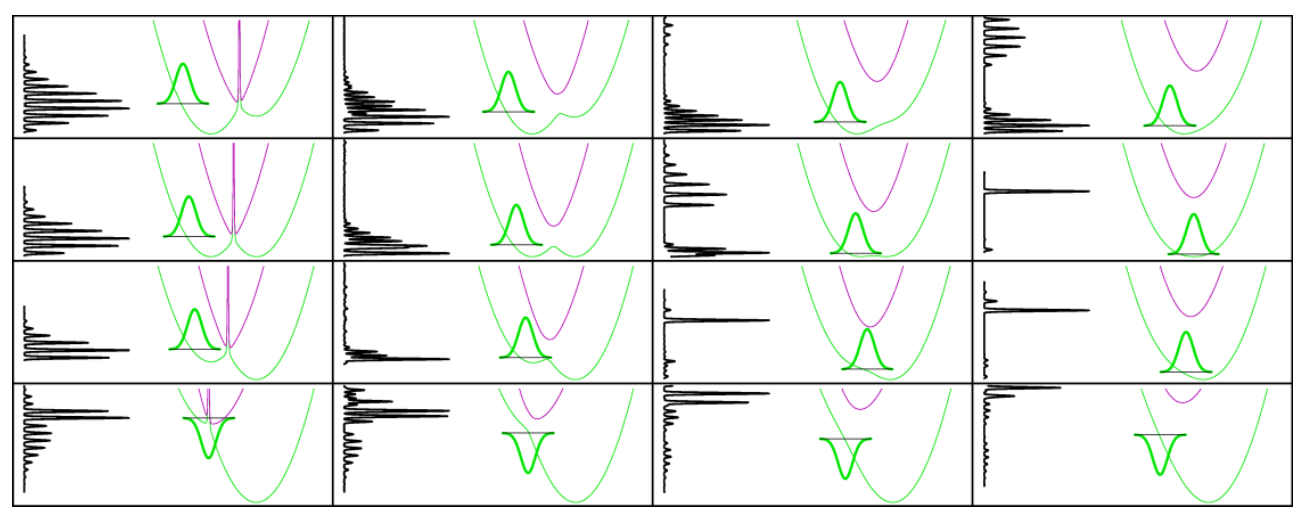

